\title{
Mixed spin ladders with exotic ground states
}

\author{
A. K. Kolezhuk \\ Institut für Theoretische Physik, Universität Hannover, Appelstr. 2, 30167 Hannover, Germany \\ and Institute of Magnetism, National Academy of Sciences and Ministry of Education of Ukraine, \\ 36(b) Vernadskii avenue, 252142 Kiev, Ukraine \\ H.-J. Mikeska \\ Institut für Theoretische Physik, Universität Hannover, Appelstr. 2, 30167 Hannover, Germany
}

(November 25, 1997)

\begin{abstract}
We study the "mixed spin" isotropic ladder system having $S=1$ spins on one leg and $S=\frac{1}{2}$ spins on the other, with general-type exchange interactions between spins on neighboring rungs. A set of model Hamiltonians with exact ground states in the form of a certain matrix product wave function is obtained. We show that sufficiently strong frustration can lead to exotic singlet ground states with infinite (exponential) degeneracy. We also list a couple of rather simple models with nontrivial ground states, including a model with only bilinear exchange.
\end{abstract}

75.10.Jm, 75.50.Ee, $75.30 . \mathrm{Kz}$

\section{INTRODUCTION}

During the last few years, "mixed" one-dimensional (1d) quantum spin systems composed of two or more different kinds of spin have drawn certain interest. Several Bethe-ansatz solvahl models with singlet ground states have been found 1 texperimentally relevant models (see, e.g Ref. 8) of ferrimagnetic quantum chains were studied 11 via various numericaland analytical approaches; recently, mixed spin chains 1213 and ladders 14 with singlet ground states were analyzed both numerically and by means of the nonlinear sigma model technique, and other interesting mixed-spin models with rich phase diagram were proposed and investigated.15

On the other hand, there has been a considerable progress in studying $1 \mathrm{~d}$ spin systems by meang the so-called matrix product (MP) states technique.16 $19 \mathrm{MP}$ states have proved to be a particularly useful tool for constructing new models with exact ground states: ground states of the MP type were found for a large family of spin-1 and spin- $\frac{3}{2}$ chain 1721 and spin- $\frac{1}{2}$ ladders 22,23 The simplest example of the MP state is the spin-1 valence bond state (VBS) which is the ground state of the AKLT mode 24 and is widely accepted as a convenient image of the Haldane-phase state. MP states were also successfully used for the variational study of the spound state and elementary excitations of spin chain 2527 and ladders.28.29 However, the states which can be accessed via the MP approach have finite, typically rather short, correlation length. Therefore they are usually gapped (except for the case of ferromagnetic-type situation with spontaneously broken symmetry 11.30 when the system has long-range order and is gapless due to the Goldstone modes) and cannot be applied for a description of the system behavior at any critical point.

In this paper, we study the isotropic mixed-spin system which may be viewed as a ladder composed of $S=1$ and $S=\frac{1}{2}$ legs, with general bilinear, biquadratic, and six- spin interactions between neighboring rungs, as shown in Fig. 1. The model is described by the Hamiltonian $\widehat{H}=\sum_{n} \widehat{h}_{n, n+1}$, where $\widehat{h}$ is chosen in the following form:

$$
\begin{aligned}
\widehat{h}_{12} & =J_{S}\left(\boldsymbol{S}_{1} \boldsymbol{S}_{2}\right)+J_{\tau}\left(\boldsymbol{\tau}_{1} \boldsymbol{\tau}_{2}\right) \\
& +\frac{1}{2} J_{r}\left(\boldsymbol{S}_{1} \boldsymbol{\tau}_{1}\right)+\frac{1}{2} J_{r}^{\prime}\left(\boldsymbol{S}_{2} \boldsymbol{\tau}_{2}\right)+J_{d}\left(\boldsymbol{\tau}_{1} \boldsymbol{S}_{2}\right)+J_{d}^{\prime}\left(\boldsymbol{S}_{1} \boldsymbol{\tau}_{2}\right) \\
& +K_{S}\left(\boldsymbol{S}_{1} \boldsymbol{S}_{2}\right)^{2}+K_{S \tau}\left(\boldsymbol{S}_{1} \boldsymbol{S}_{2}\right)\left(\boldsymbol{\tau}_{1} \boldsymbol{\tau}_{2}\right) \\
& +K_{r r}\left(\boldsymbol{S}_{1} \boldsymbol{\tau}_{1}\right)\left(\boldsymbol{S}_{2} \boldsymbol{\tau}_{2}\right)+K_{d d}\left(\boldsymbol{\tau}_{1} \boldsymbol{S}_{2}\right)\left(\boldsymbol{S}_{1} \boldsymbol{\tau}_{2}\right) \\
& +K_{r d}\left(\boldsymbol{S}_{1} \boldsymbol{\tau}_{1}\right)\left(\boldsymbol{S}_{1} \boldsymbol{\tau}_{2}\right)+K_{r d}^{\prime}\left(\boldsymbol{S}_{2} \boldsymbol{\tau}_{2}\right)\left(\boldsymbol{\tau}_{1} \boldsymbol{S}_{2}\right) \\
& +\left(\boldsymbol{S}_{1} \boldsymbol{S}_{2}\right)\left[K_{1}\left(\boldsymbol{S}_{1} \boldsymbol{\tau}_{1}\right)+K_{1}^{\prime}\left(\boldsymbol{S}_{2} \boldsymbol{\tau}_{2}\right)\right] \\
& +\left(\boldsymbol{S}_{1} \boldsymbol{S}_{2}\right)\left[K_{2}\left(\boldsymbol{\tau}_{1} \boldsymbol{S}_{2}\right)+K_{2}^{\prime}\left(\boldsymbol{S}_{1} \boldsymbol{\tau}_{2}\right)\right] \\
& +U_{1}\left(\boldsymbol{S}_{1} \boldsymbol{S}_{2}\right)^{2}\left(\boldsymbol{\tau}_{1} \boldsymbol{\tau}_{2}\right)+U_{2}\left(\boldsymbol{S}_{1} \boldsymbol{\tau}_{1}\right)\left(\boldsymbol{S}_{1} \boldsymbol{S}_{2}\right)\left(\boldsymbol{S}_{2} \boldsymbol{\tau}_{2}\right) \\
& +U_{3}\left(\boldsymbol{\tau}_{1} \boldsymbol{S}_{2}\right)\left(\boldsymbol{S}_{1} \boldsymbol{S}_{2}\right)\left(\boldsymbol{S}_{1} \boldsymbol{\tau}_{2}\right)-E_{0} \cdot \widehat{1}
\end{aligned}
$$

Here $\boldsymbol{S}$ and $\boldsymbol{\tau}$ denote spin-1 and spin- $\frac{1}{2}$ operators, respectively, and symmetric ordering of spin-1 operators is assumed wherever it is necessary.

We construct different singlet MP wave functions interpolating between a few simple VBS states, and use the technique of "optimal ground states" 11 to find a family of Hamiltonians for which those wave functions are exact ground states. Among the members of this family, the following interesting representatives are found:

(i) "AKLT-like" models which differ from the AKLT model by a few additional terms;

(ii) biquadratically coupled chains which do not contain bilinear exchange interactions between the $S=1$ and $S=\frac{1}{2}$ legs;

(iii) a model with purely bilinear interactions;

(iv) multicritical models with infinitely degenerate singlet ground states (for finite systems the degeneracy is exponentially large).

By the construction of our MP ansatz, all the ground states are dimerized, and since we choose the initial Hamiltonian to be translationally invariant, they are 
spontaneously dimerized. This is in line with the recent field-theoretical argument 31 that sufficiently strong biquadratic interaction can cause spontaneous dimerization in $S=\frac{1}{2}$ ladder, implying also a similar two-particle structure of the spectrum ("absence of magnons"). [The elementary excitation of a spontaneously dimerized system is a pair of domain walls in the dimer order.]

The paper is organized as follows: Sect. II explains the principles of constructing the matrix product ansatz and the procedure of finding the set of exact solutions, Sect. III contains the most important results describing representative models as listed above, and Sect. IV gives a brief summary. For convenience, technical details and general solutions are listed in the Appendices, so that those readers who are not interested in the details may go directly to Sect. III.

\section{THE MATRIX PRODUCT ANSATZ AND OPTIMAL GROUND STATES CONSTRUCTION}

We construct the MP ansatz for the model (11) from rectangular matrices $g^{L}$ and $g^{R}$ as follows:

$$
\left|\Psi_{0}\right\rangle=\operatorname{Tr}\left(g_{1}^{L} g_{2}^{R} g_{3}^{L} g_{4}^{R} \cdots g_{2 N-1}^{L} g_{2 N}^{R}\right),
$$

where the matrix $g_{i}^{L, R}$ contains spin states of the $i$-th rung only, and the total number of rungs is $2 N$. We demand that $\left|\Psi_{0}\right\rangle$ is a global singlet, then according to the approach proposed in Ref. 11 this dictates the following structure of the elementary matrices $g^{L, R}$ :

$$
\begin{aligned}
g^{L, R} & =\sum_{k, \lambda=\frac{1}{2}, \frac{3}{2}} \sum_{q, \mu} c^{(k, \lambda)}\langle 00 \mid k q, \lambda \mu\rangle T_{L, R}^{k q}\left|\psi_{\lambda \mu}\right\rangle \\
& =a \frac{1}{\sqrt{2}}\left(T_{L, R}^{\frac{1}{2}, \frac{1}{2}}|\Downarrow\rangle-T_{L, R}^{\frac{1}{2},-\frac{1}{2}}|\Uparrow\rangle\right) \\
& +b \frac{1}{2}\left(T_{L, R}^{\frac{3}{2}, \frac{3}{2}}|\overline{3}\rangle-T_{L, R}^{\frac{3}{2},-\frac{3}{2}}|3\rangle-T_{L, R}^{\frac{3}{2}, \frac{1}{2}}|\overline{1}\rangle+T_{L, R}^{\frac{3}{2},-\frac{1}{2}}|1\rangle\right) .
\end{aligned}
$$

Here $T_{L, R}^{k q}$ are in general arbitrary matrix representations of irreducible tensor operators $\widehat{T}^{k q}$ transforming under rotations $\widehat{R}$ according to the $\mathcal{D}^{k}$ representation of the rotation group:

$$
\widehat{R} \widehat{T}^{k q}=\sum_{q^{\prime}} \mathcal{D}_{q^{\prime} q}^{k}(\widehat{R}) \widehat{T}^{k q^{\prime}},
$$

and $\left|\psi_{\lambda \mu}\right\rangle$ are the rung wave functions with total spin $\lambda$ and its $z$-projection $\mu$. The quantities $a \equiv c^{\left(\frac{1}{2}, \frac{1}{2}\right)}$ and $b \equiv c^{\left(\frac{3}{2}, \frac{3}{2}\right)}$ are free parameters. We use the compact notation $|\Uparrow\rangle,|\Downarrow\rangle$ for the rung states having $\lambda=\frac{1}{2}$, and $|1\rangle,|\overline{1}\rangle,|3\rangle,|\overline{3}\rangle$ for the states with $\operatorname{spin} \lambda=\frac{3}{2}$ :

$$
\begin{aligned}
& |\Uparrow\rangle=\sqrt{\frac{2}{3}}|+\downarrow\rangle-\frac{1}{\sqrt{3}}|0 \uparrow\rangle, \quad|\downarrow\rangle=\frac{1}{\sqrt{3}}|0 \downarrow\rangle-\sqrt{\frac{2}{3}}|-\uparrow\rangle, \\
& |3\rangle=|+\uparrow\rangle, \quad|\overline{3}\rangle=|-\downarrow\rangle, \\
& |1\rangle=\sqrt{\frac{2}{3}}|0 \uparrow\rangle+\frac{1}{\sqrt{3}}|+\downarrow\rangle, \quad|\overline{1}\rangle=\sqrt{\frac{2}{3}}|0 \downarrow\rangle+\frac{1}{\sqrt{3}}|-\uparrow\rangle ;
\end{aligned}
$$

here single arrows $|\uparrow\rangle,|\downarrow\rangle$ indicate the spin- $\frac{1}{2}$ states, and $|+\rangle,|0\rangle,|-\rangle$ denote spin-1 states.

The matrix elements of any irreducible tensor operator (for definiteness, let us choose $\widehat{T}_{L}^{k q}$ ) in a fixed basis, according to the Wigner-Eckart theorem, are given by

$$
\begin{aligned}
T_{L}^{k q}\left(M^{\prime}, M\right) & \equiv\left\langle J M\left|\widehat{T}_{L}^{k q}\right| J^{\prime} M^{\prime}\right\rangle \\
& =\widetilde{T}_{J, J^{\prime}}^{k}\left\langle J M \mid k q, J^{\prime} M^{\prime}\right\rangle,
\end{aligned}
$$

where the reduced matrix element $\widetilde{T}_{J, J^{\prime}}^{k}$ does not depend on $M, M^{\prime}, q$ and thus can be absorbed into the free parameters $c^{(k \lambda)}$ in (3). We need $k$ to be halfinteger, therefore necessarily $J \neq J^{\prime}$ and one arrives at a $(2 J+1) \times\left(2 J^{\prime}+1\right)$ non-square matrix. Further, we fix the choice of $\widehat{T}_{R}^{k q}$ defining it as

$$
\left(\widehat{T}_{L}^{k q}\right)^{\dagger}=(-)^{k-q} \widehat{T}_{R}^{k,-q}
$$

For our problem one can choose, e.g., $J=1$ and $J^{\prime}=\frac{1}{2}$, then we have $3 \times 2$ and $2 \times 3$ matrices for $T_{L}$ and $T_{R}$, respectively, so that the dimension of matrix space coincides with the total number of states of one rung.

More complicated basis for $T_{L}^{k q}$ can be chosen, e.g., one may "decompose" $J=1$ into two $J_{1}=J_{2}=\frac{1}{2}$ and define

$$
\begin{array}{r}
T_{L}^{k q, J_{12}}\left(M^{\prime}, M_{1} M_{2}\right) \equiv\left\langle J_{1} M_{1} J_{2} M_{2}\left|T_{L}^{k q}\right| J_{12} J M\right\rangle \\
=\widetilde{T}_{J, J_{1}, J_{2}, J_{12}}^{k}\left\langle J_{12} J M \mid k q, J_{1} M_{1}, J_{2} M_{2}\right\rangle,
\end{array}
$$

where $J_{12}$ denotes the eigenvalues of the operator $\left(\widehat{\mathbf{J}}_{1}+\right.$ $\left.\widehat{\mathbf{J}}_{2}\right)^{2}$. Then, combining $M_{1}$ and $M_{2}$ into one "composite" index, one gets $4 \times 2$ matrices for $T_{L}^{k q}$ and $4 \times 2$ respectively for $T_{R}^{k q}$. Another difference with the previous case is that now for $k=\frac{1}{2}$ there are two possibilities: $J_{12}=1$ or 0 , and the set of matrices $T^{\frac{1}{2} q}$ acquires an additional free parameter $w$ :

$$
T_{L, R}^{\frac{1}{2} q}=T_{L, R}^{\frac{1}{2} q, 1}+w T_{L, R}^{\frac{1}{2} q, 0}
$$

For the sake of brevity, we will further refer to those two construction as " $2 \times 3$ " and " $2 \times 4$ " MP ansätze; the explicit form of the matrices we used can be found in the Appendix A [see Eqs. A1 and (A2)]. One may think of the above construction as of interpolating between several VBS states shown in Fig. 2. It is easy to check, for example, that the $2 \times 4$ ansatz at $(b / a=\sqrt{2}, w=0)$ and $2 \times 3$ ansatz at $b / a=\sqrt{2}$ correspond to the same state with completely dimerized legs shown in Fig. 2a, another choice of parameters $(a / b=-2 \sqrt{2}, w= \pm \sqrt{3})$ leads to the state with $S=\frac{1}{2}$ leg dimerized and $S=1$ leg in the AKLT-type VBS state (Fig. 2pb), and, finally, the combinations $(w= \pm 1 / \sqrt{3}, b=0)$ and $(b=0, a \rightarrow 0$, 
$w \propto 1 / a)$ give two "U-shape" dimerized states shown in Fig. 2c,d respectively.

Our procedure of constructing the exact ground states follows the ideas presented in Refs. 17, 19, 21: we require the MP wave function (2) to be a zero-energy groundstate of the local Hamiltonian $\widehat{h}_{i, i+1}$, which ensures that it is a ground state of the global Hamiltonian $\widehat{H}$ (an $o p$ timal ground state, in the terminology of Ref. 21). This yields the following conditions:

(i) $\widehat{h}_{i, i+1}$ annihilates all states being matrix elements of the two products $g_{i}^{L} g_{i+1}^{R}$ and $g_{i}^{R} g_{i+1}^{L}$ :

$$
\widehat{h}_{12}\left(g_{1}^{L} g_{2}^{R}\right)=0, \quad \widehat{h}_{12}\left(g_{1}^{R} g_{2}^{L}\right)=0 ;
$$

(ii) all other eigenstates of $\widehat{h}_{12}$ have the energy $\varepsilon \geq 0$. Then $\left|\Psi_{0}\right\rangle$ is the zero-energy ground state of $\widehat{H}$; if one drops the constant term $-E_{0} \cdot \hat{1}$ in (1), the remaining Hamiltonian has the energy density $E_{0}$ per rung.

For further treatment it is convenient to write the Hamiltonian $\widehat{h}$ in terms of projectors on the states $\left|\Psi_{J M}^{(k)}\right\rangle$ of the two-rung plaquette $(i, i+1)$ with fixed angular momentum. The complete set of the plaquette states [see Eqs. (A3)] contains one multiplet with $J=3$, three quintuplets, four triplets and two singlets, and thus one obtains:

$$
\begin{aligned}
& \widehat{h}=\sum_{k, l=1,2} \lambda_{0}^{(k, l)}\left|\Psi_{00}^{(k)}\right\rangle\left\langle\Psi_{00}^{(l)}\left|+\sum_{k, l=1 . .4} \lambda_{1}^{(k, l)} \sum_{M}\right| \Psi_{1 M}^{(k)}\right\rangle\left\langle\Psi_{1 M}^{(l)}\right| \\
& +\sum_{k, l=1 . .3} \lambda_{2}^{(k, l)} \sum_{M}\left|\Psi_{2 M}^{(k)}\right\rangle\left\langle\Psi_{2 M}^{(l)}\left|+\lambda_{3} \sum_{M}\right| \Psi_{3 M}\right\rangle\left\langle\Psi_{3 M}\right| .
\end{aligned}
$$

Here obviously $\lambda_{J}^{(k, l)}=\left(\lambda_{J}^{(l, k)}\right)^{*}$ because of the hermitian property of $\widehat{h}$. The complete set of the plaquette states can be divided into two subsets: local ground states $\left|\Psi_{J M}^{g,(k)}\right\rangle, k=1, \ldots n_{J}^{(g)}$ which are contained in the matrix products $g^{L} g^{R}$ and $g^{R} g^{L}$, and local eigenstates $\left|\Psi_{J M}^{e,(k)}\right\rangle$, $k=1, \ldots n_{J}^{(e)}$ which do not enter there.

The conditions (i) mean that the local Hamiltonian $\widehat{h}$ should project only onto the states $\left|\Psi_{J M}^{e,(k)}\right\rangle$, and the multiplets $\left|\Psi_{J M}^{g,(k)}\right\rangle$ have to be absent in Eq. (9). This results in the following system of equations:

$$
\begin{array}{ll}
\lambda_{J}^{(g,(k) ; g,(l))}=0, & k=1 \ldots n_{J}^{(g)}, \quad l=k \ldots n_{J}^{(g)} \\
\lambda_{J}^{\left(e,\left(k^{\prime}\right) ; g,(k)\right)}=0, & k=1 \ldots n_{J}^{(g)}, \quad k^{\prime}=1 \ldots n_{J}^{(e)}
\end{array}
$$

which is essentially a system of linear equations in the Hamiltonian coupling constants $J_{. .}, K_{. .}, U_{\text {.. [ }}$ [see Eq. (11)].

The conditions (ii) require that all the eigenstates within the subspace determined by the basis $\left|\Psi_{J M}^{e,(k)}\right\rangle$ have positive energy, which yields the inequalities

$$
\widetilde{\lambda}_{J}^{(\alpha)} \geq 0, \quad \alpha=1 \ldots n_{J}^{(e)},
$$

where $\widetilde{\lambda}_{J}^{(\alpha)}$ denotes the eigenvalues of the matrix

$$
\left[\begin{array}{lll}
\lambda_{J}^{(1,1)} & \cdots & \lambda_{J}^{\left(1, n_{J}^{(e)}\right)} \\
\vdots & \ddots & \vdots \\
\lambda_{J}^{\left(n_{J}^{(e)}, 1\right)} & \cdots & \lambda_{J}^{\left(n_{J}^{(e)}, n_{J}^{(e)}\right)}
\end{array}\right]
$$

If one or more of $\widetilde{\lambda}_{J}^{(\alpha)}$ is zero, this may indicate an additional degeneracy of the ground state.

Eqs. (3), (5), (6), (7) and (8), (10), (11) will be the basis for the further analysis. Now we proceed to considering specific solutions of those equations in various particular cases.

\section{MODELS WITH EXACT GROUND STATES}

In this section we present a number of models with exact ground states being the most simple representatives of different classes of solutions mentioned in the Introduction.

\section{A. $2 \times 3$ MP ansatz}

\section{General case}

It is easy to verify that for the $2 \times 3 \mathrm{MP}$ ansatz the two matrix products $g^{L} g^{R}$ and $g^{R} g^{L}$ contain generally only the following multiplets:

$$
\begin{aligned}
& a^{2} \psi_{00}^{11}+\left(b^{2} / \sqrt{2}\right) \psi_{00}^{33} \equiv\left|\Psi_{00}^{(2)}\right\rangle, \\
& \psi_{1 M}^{11} \equiv\left|\Psi_{1 M}^{(2)}\right\rangle, \quad \psi_{1 M}^{33} \equiv\left|\Psi_{1 M}^{(3)}\right\rangle, \\
& (1 / \sqrt{2})\left(\psi_{1 M}^{31}+\psi_{1 M}^{13}\right) \equiv\left|\Psi_{1 M}^{(2)}\right\rangle, \\
& b^{2} \psi_{2 M}^{33}+\sqrt{2} a b\left(\psi_{2 M}^{31}-\psi_{2 M}^{13}\right) \equiv\left|\Psi_{2 M}^{(3)}\right\rangle,
\end{aligned}
$$

then the remaining multiplets can be chosen as

$$
\begin{aligned}
& \left|\Psi_{00}^{(1)}\right\rangle=\left(b^{2} / \sqrt{2}\right) \psi_{00}^{11}-a^{2} \psi_{00}^{33}, \\
& \left|\Psi_{1 M}^{(1)}\right\rangle=\frac{1}{\sqrt{2}}\left(\psi_{1 M}^{31}-\psi_{1 M}^{13}\right),\left|\Psi_{2 M}^{(1)}\right\rangle=\frac{1}{\sqrt{2}}\left(\psi_{2 M}^{31}+\psi_{2 M}^{13}\right), \\
& \left|\Psi_{2 M}^{(2)}\right\rangle=2 a b \psi_{2 M}^{33}-\left(b^{2} / \sqrt{2}\right)\left(\psi_{2 M}^{31}-\psi_{2 M}^{13}\right) .
\end{aligned}
$$

The conditions (10,11) now take the form

$$
\begin{aligned}
& \lambda_{0}^{(2,2)}=\lambda_{0}^{(1,2)}=\lambda_{0}^{(2,1)}=0, \\
& \lambda_{1}^{(1, k)}=\lambda_{1}^{(k, l)}=0, \quad 2 \leq k \leq 4, \quad k \leq l \leq 4, \\
& \lambda_{2}^{(1,3)}=\lambda_{2}^{(2,3)}=\lambda_{2}^{(3,3)}=0, \\
& \lambda_{0}^{(1,1)} \geq 0, \lambda_{1}^{(1,1)} \geq 0, \quad \lambda_{3} \geq 0, \quad \tilde{\lambda}_{2}^{\alpha} \geq 0,
\end{aligned}
$$

where $\widetilde{\lambda}_{2}^{\alpha}, \alpha=1,2$ are the eigenvalues of the matrix

$$
\left[\begin{array}{ll}
\lambda_{2}^{(1,1)} & \lambda_{2}^{(1,2)} \\
\lambda_{2}^{(2,1)} & \lambda_{2}^{(2,2)}
\end{array}\right] .
$$


For the sake of simplicity we now set $\lambda_{2}^{(1,2)}=0$. In fact, one can show that this requirement just fixes certain "natural" symmetries in (1), namely,

$$
J_{r}=J_{r}^{\prime}, \quad J_{d}=J_{d}^{\prime}, \quad K_{r d}=K_{r d}^{\prime}, \quad K_{1,2}=K_{1,2}^{\prime} .
$$

Further, we require the six-spin couplings in (11) to be zero, in order to make the model less cumbersome. Then one obtains the sytem of nineteen equations [fifteen equations (14) and four additional assumptions $\lambda_{2}^{(1,2)}=0$, $\left.U_{1,2,3}=0\right]$ for twenty parameters of the Hamiltonian (1) and the free parameter $u=a / b$ entering the $2 \times 3 \mathrm{MP}$ ansatz (3), (5). It turns out that one of those nineteen equations is linearly dependent, and the general solution contains, beside $u$, two additional free parameters $x$ and $y$. This solution in its general form is presented in Appendix B [see Eqs. (B1), (B2)], and here we will just consider its most interesting particular cases:

(a) Setting $u=-\frac{1}{4} \sqrt{2}, x=\frac{32}{27} y$ and fixing the energy scale by choosing $y=\frac{1}{3}$, one gets the "AKLT-type" model of the form

$$
\begin{aligned}
\widehat{H} & =\sum_{n} \boldsymbol{S}_{n} \boldsymbol{S}_{n+1}+\frac{1}{3}\left(\boldsymbol{S}_{n} \boldsymbol{S}_{n+1}\right)^{2} \\
& +\frac{1}{3} \boldsymbol{S}_{n}\left(2 \boldsymbol{\tau}_{n}+\boldsymbol{\tau}_{n-1}+\boldsymbol{\tau}_{n+1}\right) \\
& +\frac{1}{3}\left(\boldsymbol{S}_{n} \boldsymbol{S}_{n+1}\right)\left[\left(\boldsymbol{S}_{n}+\boldsymbol{S}_{n+1}\right) \cdot\left(\boldsymbol{\tau}_{n}+\boldsymbol{\tau}_{n+1}\right)\right],
\end{aligned}
$$

with the energy density per rung $E_{0}=-2 / 3$. (We recall that symmetric ordering of spin- 1 operators is implicitly assumed). For this model two more eigenvalues of the local Hamiltonian are zero:

$$
\lambda_{0}=\lambda_{1}=0,
$$

which may in principle indicate higher degeneracy of the ground state (there always exists another dimerized singlet state which can be obtained by the translation in one rung, and $\lambda_{1}=0$ may mean degeneracy with some "partially ferromagnetic" state with the total spin $J=1$ of each plaquette).

(b) Setting $u=-\frac{1}{2} \sqrt{2}, y=0$, and choosing $x=\frac{4}{9}$ to fix the scale, one obtains a model of $S=1$ and $S=\frac{1}{2}$ chains coupled with purely biquadratic interaction:

$$
\begin{aligned}
\widehat{H} & =\sum_{n} \boldsymbol{S}_{n} \boldsymbol{S}_{n+1}+\frac{8}{3} \boldsymbol{\tau}_{n} \boldsymbol{\tau}_{n+1} \\
& +\left(\boldsymbol{S}_{n} \boldsymbol{S}_{n+1}\right)\left[\boldsymbol{\tau}_{n} \boldsymbol{\tau}_{n+1}+\left(\boldsymbol{S}_{n}+\boldsymbol{S}_{n+1}\right) \cdot\left(\boldsymbol{\tau}_{n}+\boldsymbol{\tau}_{n+1}\right)\right]
\end{aligned}
$$

with the energy per rung $E_{0}=-2$. For this model also one of the local Hamiltonian eigenvalues vanishes,

$$
\lambda_{2}^{(1,1)}=0 .
$$

Another model of this type can be obtained by setting $u=-\frac{1}{4} \sqrt{2}, y=0$; after choosing $x=\frac{8}{27}$ to fix a proper scale the Hamiltonian takes the form

$$
\begin{aligned}
\widehat{H} & =\sum_{n} \boldsymbol{S}_{n} \boldsymbol{S}_{n+1}+2 \boldsymbol{\tau}_{n} \boldsymbol{\tau}_{n+1}+\left(\boldsymbol{S}_{n} \boldsymbol{S}_{n+1}\right)^{2} \\
& +\frac{1}{4}\left(\boldsymbol{S}_{n} \boldsymbol{S}_{n+1}\right)\left[\left(\boldsymbol{S}_{n}+\boldsymbol{S}_{n+1}\right) \cdot\left(\boldsymbol{\tau}_{n}+\boldsymbol{\tau}_{n+1}\right)\right] \\
& -\left[\left(\boldsymbol{S}_{n}+\boldsymbol{S}_{n+1}\right) \cdot \boldsymbol{\tau}_{n}\right]\left[\left(\boldsymbol{S}_{n}+\boldsymbol{S}_{n+1}\right) \cdot \boldsymbol{\tau}_{n+1}\right] .
\end{aligned}
$$

The energy per rung is $E_{0}=-1$, and the eigenvalue $\lambda_{0}=0$.

(c) If $x=0$, then one can somewhat surprisingly observe that the solution (B1) does not depend on the parameter $u=a / b$ entering the MP wavefunction (3). Three of the local Hamiltonian eigenvalues are now zero,

$$
\lambda_{0}=0, \quad \lambda_{2}^{(1,1)}=0, \quad \lambda_{3}=0,
$$

and one has to put $y<0$ for the remaining ones to be positive. Setting the energy scale by fixing $y=-\frac{1}{8}$, one obtains the model

$$
\begin{aligned}
\widehat{H} & =\sum_{n} \boldsymbol{\tau}_{n} \boldsymbol{\tau}_{n+1}+\frac{1}{8} \boldsymbol{S}_{n} \boldsymbol{S}_{n+1} \\
& -\frac{1}{8} \boldsymbol{S}_{n}\left(2 \boldsymbol{\tau}_{n}+\boldsymbol{\tau}_{n-1}+\boldsymbol{\tau}_{n+1}\right)+\frac{1}{2}\left(\boldsymbol{S}_{n} \boldsymbol{S}_{n+1}\right)\left(\boldsymbol{\tau}_{n} \boldsymbol{\tau}_{n+1}\right) \\
& -\frac{1}{2}\left[\left(\boldsymbol{S}_{n}+\boldsymbol{S}_{n+1}\right) \cdot \boldsymbol{\tau}_{n}\right]\left[\left(\boldsymbol{S}_{n}+\boldsymbol{S}_{n+1}\right) \cdot \boldsymbol{\tau}_{n+1}\right]
\end{aligned}
$$

with the energy density $E_{0}=-\frac{1}{4}$ per rung. The fact that $\lambda_{3}=0$ means that the singlet ground state of the model is degenerate with the fully polarized ferromagnetic state: the ferromagnetic state is the eigenstate of the Hamiltonian, and it is a straightforward exercise to check that it has the same energy. However, the ground state degeneracy of the model (19) is much higher: any wave function $|\Psi(u)\rangle$ of the form (2), (3) with arbitrary parameter $u$ is the ground state. One can easily calculate the overlap between two such wave functions having different values of $u$ :

$$
\left\langle\Psi\left(u_{1}\right) \mid \Psi\left(u_{2}\right)\right\rangle=q^{N}, \quad q=\frac{\left(1+u_{1} u_{2}\right)^{2}}{\left(1+u_{1}^{2}\right)\left(1+u_{2}^{2}\right)} \leq 1,
$$

i.e., the two g.s. wave functions with different values of $u$ are asymptotically orthogonal in thermodynamic limit $N \rightarrow \infty$ with the overlap vanishing exponentially with the increase of $N$. This means that the dimension of the basis of this subspace $\{|\Psi(u)\rangle\}$, i.e., the number of mutually orthogonal ground states, is exponentially large in thermodynamic limit. This is an example of multicritical model. Unfortunately, within the present approach one cannot make any statement about presence of the gap. It is worthwhile to mention that infinitely degenerate ground state in a mixed spin- $1 /$ spin- $\frac{1}{2}$ system was observed in certain limiting case of de Vega-Woynarovich model (see the discussion of $\bar{c}=0$ case in Ref. 3) when the velocity of one of the two spinon branches with linear dispersion becomes zero; however, since a pair of spinons can be combined either in a singlet or in a triplet with the same energy, the set of degenerate ground states in that case should contain not only singlets, but states of higher spin as well. 


\section{Case $b=0$ (no spin- $\frac{3}{2}$ states on the rung)}

For $2 \times 3 \mathrm{MP}$ ansatz it is useful to consider separately the case $b=0$, because it turns out to lead to a new type of solution. One can see that at $b=0$ the spin- $\frac{3}{2}$ states of the ladder rungs are excluded from the wavefunction (3), and only the following two multiplets are present in the matrix products $g_{1}^{L} g_{2}^{R}, g_{1}^{R} g_{2}^{L}$ :

$$
\left|\Psi_{00}^{g}\right\rangle=\left|\psi_{00}^{11}\right\rangle, \quad\left|\Psi_{1 M}^{g}\right\rangle=\left|\psi_{1 M}^{11}\right\rangle .
$$

It turns out that in this case it is possible to obtain a nontrivial solution of the system (10) with only bilinear coupling, which correspond to the following model:

$$
\begin{aligned}
\widehat{H} & =\sum_{n} \gamma \boldsymbol{S}_{n} \boldsymbol{\tau}_{n}-\boldsymbol{\tau}_{n} \boldsymbol{\tau}_{n+1}-\boldsymbol{S}_{n} \boldsymbol{S}_{n+1} \\
& -\boldsymbol{S}_{n}\left(\boldsymbol{\tau}_{n-1}+\boldsymbol{\tau}_{n+1}\right) .
\end{aligned}
$$

Here $\gamma \geq \frac{4}{3}$ is an arbitrary parameter, and the energy density is $E_{0}=-\left(\gamma+\frac{1}{4}\right)$ per rung. At $\gamma=\frac{4}{3}$ the eigenvalue $\lambda_{3}$ vanishes, indicating the first-order transition into fully polarized ferromagnetic state [cf. a similar transition for $S=\frac{1}{2}$ ladder in Ref. 23]. The matrix product $g_{1}^{L} g_{2}^{R}$ has the simple form

$$
\left[\begin{array}{cc}
2|\Uparrow \Downarrow\rangle-|\Downarrow \Uparrow\rangle & |\downarrow \Downarrow \downarrow\rangle \\
-|\Uparrow \Uparrow\rangle & -2|\Downarrow \Uparrow\rangle+|\Uparrow \Downarrow\rangle
\end{array}\right]
$$

which allows one to "visualize" the structure of the ground state as interpolating between two "U-shape" VBS states shown in Fig. 2 2 ,d [here $\Uparrow, \Downarrow$ are the rung states with total spin $\frac{1}{2}$, see Eqs. (4)].

\section{B. $2 \times 4$ MP ansatz}

For the $2 \times 4 \mathrm{MP}$ ansatz, at a general choice of parameters $a, b, w$ entering the wave function (3), the following multiplets are contained in the two matrix products $g^{L} g^{R}$ and $g^{R} g^{L}$ :

$$
\begin{aligned}
& \psi_{00}^{11}, \quad \psi_{00}^{33}, \\
& \psi_{1 M}^{11}, \quad \psi_{1 M}^{33}, \psi_{1 M}^{13}, \quad \psi_{1 M}^{31}, \\
& (1 / \sqrt{2})\left(\psi_{2 M}^{31}-\psi_{2 M}^{13}\right), \quad \psi_{2 M}^{33} .
\end{aligned}
$$

We require them to be annihilated by the local Hamiltonian $\widehat{h}$, and the remaining multiplets

$$
(1 / \sqrt{2})\left(\psi_{2 M}^{31}-\psi_{2 M}^{13}\right), \quad \psi_{3 M}^{33}
$$

to be the eigenstates of $\widehat{h}$ with positive energy. Eqs. (10) give a system of eighteen linear equations for twenty parameters of the Hamiltonian (1), and its general solution contains two free parameters $x, y$ (one of them is again irrelevant since it just sets the energy scale). The multiplets (24) do not contain the wave function parameters $a, b, w$, and thus the solution also does not depend on them, yielding a one-parametric family of multicritical models with infinitely degenerate ground state similar to one discussed in the previous subsection. The solution is given by

$$
\begin{aligned}
& J_{r}=J_{r}^{\prime}=2 x, \quad J_{d}=J_{d}^{\prime}=K_{1}=K_{1}^{\prime}=K_{2}=K_{2}^{\prime}=x, \\
& J_{S}=\frac{3}{2} y, \quad J_{\tau}=\frac{4}{7}(5 y-4 x), \\
& K_{S}=\frac{1}{2} y, \quad K_{S \tau}=\frac{2}{7}(13 y-9 x), \\
& K_{r r}=K_{d d}=3 x-2 y, \quad K_{r d}=K_{r d}^{\prime}=\frac{4}{7}(3 x-2 y), \\
& U_{1}=\frac{2}{7}(5 y-4 x), \quad U_{2}=U_{3}=\frac{4}{7}(3 x-2 y),
\end{aligned}
$$

and the conditions (11) take the form

$$
\lambda_{2}=8(y-x) \geq 0, \quad \lambda_{3}=\frac{20}{7}(2 x+y) \geq 0 .
$$

This class of solutions is more cumbersome than in case of the $2 \times 3 \mathrm{MP}$ ansatz; for instance, one may observe that six-spin interactions $U_{1,2,3}$ in (26) should be always nonzero. The simplest model within this class is achieved by setting $y=\frac{3}{2} x$, its Hamiltonian after fixing the proper energy scale is

$$
\begin{aligned}
\widehat{H} & =\sum_{n} \boldsymbol{S}_{n} \boldsymbol{S}_{n+1}+\frac{1}{3}\left(\boldsymbol{S}_{n} \boldsymbol{S}_{n+1}\right)^{2}\left[1+\frac{4}{3} \boldsymbol{\tau}_{n} \boldsymbol{\tau}_{n+1}\right] \\
& +\frac{8}{9} \boldsymbol{\tau}_{n} \boldsymbol{\tau}_{n+1}+\frac{4}{9} \boldsymbol{S}_{n}\left(2 \boldsymbol{\tau}_{n}+\boldsymbol{\tau}_{n-1}+\boldsymbol{\tau}_{n+1}\right) \\
& +\frac{4}{9}\left(\boldsymbol{S}_{n} \boldsymbol{S}_{n+1}\right)\left[\left(\boldsymbol{S}_{n}+\boldsymbol{S}_{n+1}\right) \cdot\left(\boldsymbol{\tau}_{n}+\boldsymbol{\tau}_{n+1}\right)\right]
\end{aligned}
$$

with the energy per rung $E_{0}=-\frac{2}{3}$. It should be remarked that, in contrast to the model (19), fully polarized ferromagnetic state generally (except for the case $\left.x=-\frac{1}{2} y\right)$ is not degenerate with the ground state of multicritical models defined by (26).

\section{SUMMARY}

We have studied the isotropic ladder composed of $S=1$ and $S=\frac{1}{2}$ chains, with general type exchange interaction between spins on neighboring rungs. The technique of matrix product states is applied to construct a family of Hamiltonians with exact ground states. Among the members of this family, we have found a couple of relatively simple models with nontrivial ground states, including one model with only bilinear exchange and two models with $S=1$ and $S=\frac{1}{2}$ chains being coupled by purely biquadratic exchange. We also present a family of multicritical models whose ground state is infinitely degenerate: in the thermodynamic limit $N \rightarrow \infty$ the number of degenerate ground states grows with $N$ exponentially. 


\section{ACKNOWLEDGMENTS}

We are grateful to H.-U. Everts and C. Waldtmann for discussion of the results. A.K. gratefully acknowledges the hospitality of Hannover Institute for Theoretical Physics during his stay there. This work was supported by the German Ministry for Research and Technology (BMBF) under the contract 03MI4HAN8 and by the Ukrainian Ministry of Science (grant 2.4/27).

\section{APPENDIX A: TECHNICAL DETAILS}

Here is the explicit form of the matrices we used in (3) for the $2 \times 3 \mathrm{MP}$ ansatz:

$$
\begin{aligned}
T_{L}^{\frac{1}{2}, \frac{1}{2}} & =\left[\begin{array}{ccc}
0 & \sqrt{\frac{1}{3}} & 0 \\
0 & 0 & \sqrt{\frac{2}{3}}
\end{array}\right], T_{L}^{\frac{1}{2},-\frac{1}{2}}=\left[\begin{array}{ccc}
-\sqrt{\frac{2}{3}} & 0 & 0 \\
0 & -\sqrt{\frac{1}{3}} & 0
\end{array}\right], \\
T_{L}^{\frac{3}{2}, \frac{3}{2}} & =\left[\begin{array}{lll}
0 & 0 & 1 \\
0 & 0 & 0
\end{array}\right], \quad T_{L}^{\frac{3}{2},-\frac{3}{2}}=\left[\begin{array}{lll}
0 & 0 & 0 \\
1 & 0 & 0
\end{array}\right], \\
T_{L}^{\frac{3}{2}, \frac{1}{2}} & =\left[\begin{array}{ccc}
0 & -\sqrt{\frac{2}{3}} & 0 \\
0 & 0 & \sqrt{\frac{1}{3}}
\end{array}\right], T_{L}^{\frac{3}{2},-\frac{1}{2}}=\left[\begin{array}{ccc}
\sqrt{\frac{1}{3}} & 0 & 0 \\
0 & -\sqrt{\frac{2}{3}} & 0
\end{array}\right] .
\end{aligned}
$$

The matrices $T_{R}$ can be easily obtained from those matrices using the definition (6).

And in case of the $2 \times 4$ ansatz the matrices were chosen as

$$
\begin{aligned}
& T_{L}^{\frac{1}{2}, \frac{1}{2}, 0}=\left[\begin{array}{cccc}
0 & 1 / \sqrt{2} & -1 / \sqrt{2} & 0 \\
0 & 0 & 0 & 0
\end{array}\right], \\
& T_{L}^{\frac{1}{2},-\frac{1}{2}, 0}=\left[\begin{array}{cccc}
0 & 0 & 0 & 0 \\
0 & 1 / \sqrt{2} & -1 / \sqrt{2} & 0
\end{array}\right], \\
& T_{L}^{\frac{1}{2}, \frac{1}{2}, 1}=\left[\begin{array}{cccc}
0 & 1 / \sqrt{6} & 1 / \sqrt{6} & 0 \\
0 & 0 & 0 & \sqrt{2 / 3}
\end{array}\right], \\
& T_{L}^{\frac{1}{2},-\frac{1}{2}, 1}=\left[\begin{array}{lccc}
-\sqrt{2 / 3} & 0 & 0 & 0 \\
0 & -1 / \sqrt{6} & -1 / \sqrt{6} & 0
\end{array}\right], \\
& T_{L}^{\frac{3}{2}, \frac{3}{2}}=\left[\begin{array}{llll}
0 & 0 & 0 & 1 \\
0 & 0 & 0 & 0
\end{array}\right], \quad T_{L}^{\frac{3}{2},-\frac{3}{2}}=\left[\begin{array}{llll}
0 & 0 & 0 & 0 \\
1 & 0 & 0 & 0
\end{array}\right], \\
& T_{L}^{\frac{3}{2}, \frac{1}{2}}=\left[\begin{array}{cccr}
0 & -1 / \sqrt{3} & -1 / \sqrt{3} & 0 \\
0 & 0 & 0 & 1 / \sqrt{3}
\end{array}\right], \\
& T_{L}^{\frac{3}{2}, \frac{1}{2}}=\left[\begin{array}{cccc}
1 / \sqrt{3} & 0 & 0 & 0 \\
0 & -1 / \sqrt{3} & -1 / \sqrt{3} & 0
\end{array}\right] \text {. }
\end{aligned}
$$

The complete set of spin states of a two-rung plaquette is, in the notation used in Eqs. (四),

$$
\begin{aligned}
& \psi_{33}^{33}=|33\rangle, \quad \psi_{3,-3}^{33}=|\overline{3} \overline{3}\rangle, \\
& \psi_{32}^{33}=\frac{1}{\sqrt{2}}(|31\rangle+|13\rangle), \quad \psi_{3,-2}^{33}=\frac{1}{\sqrt{2}}(|\overline{3} \overline{1}\rangle+|\overline{1} \overline{3}\rangle), \\
& \psi_{3,1}^{33}=(1 / \sqrt{5})(\sqrt{3}|11\rangle+|3 \overline{1}\rangle+|\overline{1} 3\rangle), \\
& \psi_{3,-1}^{33}=(1 / \sqrt{5})(\sqrt{3}|\overline{1} \overline{1}\rangle+|\overline{3} 1\rangle+|1 \overline{3}\rangle),
\end{aligned}
$$

$$
\begin{aligned}
& \psi_{30}^{33}=(1 / 2 \sqrt{5})\{|3 \overline{3}\rangle+|\overline{3} 3\rangle+3(|1 \overline{1}\rangle+|\overline{1} 1\rangle)\}, \\
& \psi_{22}^{33}=\frac{1}{\sqrt{2}}(|31\rangle-|13\rangle), \quad \psi_{2,-2}^{33}=-\frac{1}{\sqrt{2}}(|\overline{3} \overline{1}\rangle-|\overline{1} \overline{3}\rangle), \\
& \psi_{21}^{33}=\frac{1}{\sqrt{2}}(|3 \overline{1}\rangle-|\overline{1} 3\rangle), \quad \psi_{2,-1}^{33}=-\frac{1}{\sqrt{2}}(|\overline{3} 1\rangle-|1 \overline{3}\rangle), \\
& \psi_{20}^{33}=(1 / 2)(|1 \overline{1}\rangle-|\overline{1} 1\rangle+|3 \overline{3}\rangle-|\overline{3} 3\rangle), \\
& \psi_{11}^{33}=\sqrt{3 / 10}(|3 \overline{1}\rangle+|\overline{1} 3\rangle-(2 / \sqrt{3})|11\rangle), \\
& \psi_{1,-1}^{33}=\sqrt{3 / 10}(|\overline{3} 1\rangle+|1 \overline{3}\rangle-(2 / \sqrt{3})|\overline{1} \overline{1}\rangle), \\
& \psi_{10}^{33}=(1 / 2 \sqrt{5})\{3(|3 \overline{3}\rangle+|\overline{3} 3\rangle)-|1 \overline{1}\rangle-|\overline{1} 1\rangle\}, \\
& \psi_{00}^{33}=(1 / 2)(|\overline{1} 1\rangle-|1 \overline{1}\rangle+|3 \overline{3}\rangle-|\overline{3} 3\rangle), \\
& \psi_{22}^{31}=|3 \Uparrow\rangle, \quad \psi_{2,-2}^{31}=|\overline{3} \Downarrow\rangle, \\
& \psi_{21}^{31}=\frac{1}{2}|3 \Downarrow\rangle+\frac{\sqrt{3}}{2}|1 \Uparrow\rangle, \quad \psi_{2,-1}^{31}=\frac{1}{2}|\overline{3} \Uparrow\rangle+\frac{\sqrt{3}}{2}|\overline{1} \Downarrow\rangle, \\
& \psi_{20}^{31}=\frac{1}{\sqrt{2}}(|1 \Downarrow\rangle+|\overline{1} \Uparrow\rangle), \quad \psi_{11}^{31}=-\frac{1}{2}|1 \Uparrow\rangle+\frac{\sqrt{3}}{2}|3 \Downarrow\rangle, \\
& \psi_{1,-1}^{31}=\frac{1}{2}|\overline{1} \Downarrow\rangle-\frac{\sqrt{3}}{2}|\overline{3} \Uparrow\rangle, \quad \psi_{10}^{31}=\frac{1}{\sqrt{2}}(|1 \Downarrow\rangle-|\overline{1} \Uparrow\rangle), \\
& \psi_{00}^{11}=\frac{1}{\sqrt{2}}(|\Uparrow \Downarrow\rangle-|\Downarrow \Uparrow\rangle), \quad \psi_{10}^{11}=\frac{1}{\sqrt{2}}(|\Uparrow \Downarrow\rangle+|\Downarrow \Uparrow\rangle), \\
& \psi_{11}^{11}=|\Uparrow \Uparrow\rangle, \psi_{1,-1}^{11}=|\Downarrow \Downarrow\rangle .
\end{aligned}
$$

Here the superscripts of $\psi$ 's denote the total momentum $J$ of left and right rung states of which they are composed (with the shortcut convention that 3 means $J=\frac{3}{2}$ and 1 means $\frac{1}{2}$ ). The states $\psi_{j m}^{13}$ can be obtained from $\psi_{j m}^{31}$ by interchanging the left and right rung states.

\section{APPENDIX B: GENERAL SOLUTION FOR THE $2 \times 3$ MP ANSATZ}

The general solution of the system (14) with four additional conditions $\lambda_{2}^{(1,2)}=0, U_{1,2,3}=0$ has the following form:

$$
\begin{aligned}
& J_{S}=\left[15 u^{4}+37 u^{2}+\frac{5}{2}-\sqrt{2} u\left(2 u^{4}-40 u^{2}-3\right)\right] x-y, \\
& J_{\tau}=\left(112 u^{4}+176 u^{3} \sqrt{2}+192 u^{2}+44 \sqrt{2} u+14\right) x-8 y, \\
& J_{r}=2 \sqrt{2}(4 u+\sqrt{2})\left(u^{2}+1\right)\left(2 u^{2}-1\right) x+2 y, \\
& J_{d}=y, \\
& K_{S}=-\frac{1}{2} \sqrt{2}(2 u+\sqrt{2})(u+\sqrt{2}) u(-2 u+\sqrt{2})^{2} x, \\
& K_{S \tau}=4\left[13 u^{4}+25 u^{2}+3 / 2\right. \\
& \left.\quad-\sqrt{2} u\left(2 u^{4}-24 u^{2}-5\right)\right] x-4 y, \\
& K_{r r}=-\sqrt{2}\left(-4 u^{4}+11 u^{3} \sqrt{2}+21 u^{2}+7 \sqrt{2} u+5\right) \\
& \quad \times(2 u+\sqrt{2}) x+4 y \\
& K_{d d}=-\sqrt{2}\left(4 u^{4}+9 u^{3} \sqrt{2}+27 u^{2}+5 \sqrt{2} u+3\right) \\
& \quad \times(2 u+\sqrt{2}) x+4 y, \\
& K_{r d}=-4\left(5 u^{2}+\sqrt{2} u+1\right)(u+\sqrt{2})
\end{aligned}
$$




$$
\begin{gathered}
\times(2 u+\sqrt{2}) x+4 y \\
K_{1}=-(-2 u+\sqrt{2})(u+\sqrt{2})\left(u^{2}+3 \sqrt{2} u+1\right) x \\
K_{2}=\frac{1}{2} \sqrt{2}(-2 u+\sqrt{2})\left(8 u^{3}+5 u^{2} \sqrt{2}+2 u-\sqrt{2}\right) u x \\
E_{0}=-\left[44 u^{4}+68 u^{2}+9 / 2\right. \\
\left.+\sqrt{2} u\left(8 u^{4}+60 u^{2}+13\right)\right] x+2 y
\end{gathered}
$$

with the conditions on the eigenvalues (11) being

$$
\begin{aligned}
& \lambda_{0}=-6 \sqrt{2}(4 u+\sqrt{2}) x \geq 0, \\
& \lambda_{1}=\left(48 u^{4}+66 u^{3} \sqrt{2}+78 u^{2}+18 \sqrt{2} u+6\right) x-4 y \geq 0, \\
& \lambda_{2}^{(1,1)}=3 \sqrt{2}(2 u+\sqrt{2})\left(u^{2}+1\right) x \geq 0, \quad(\mathrm{~B} 1 \mathrm{~b}) \\
& \lambda_{2}^{2,2}=12\left(12 u^{3}+9 u^{2} \sqrt{2}+4 u+\sqrt{2}\right) \\
& \quad \times(u+\sqrt{2}) x-12 y \geq 0, \\
& \lambda_{3}=60 u^{2}(u+\sqrt{2})^{2} x \geq 0 .
\end{aligned}
$$

Here $u=a / b$ is the free parameter entering the $2 \times 3$ MP ansatz (3), and $x, y$ are additional free parameters arising from the solution of the linear system (14). Taking into account that one of the parameters just sets the energy scale and thus is irrelevant, one gets from (B1) a two-parameter family of Hamiltonians with exact ground states in a form of the $2 \times 3$ matrix product. One can see that inequalities $(\mathrm{B} 1 \mathrm{~b})$ can be satisfied provided that

$$
x \geq 0, \quad-\frac{\sqrt{2}}{4} \leq u \leq-\frac{\sqrt{2}}{2} .
$$

* Permanent address: Institute of Magnetism, 36(b) Vernadskii avenue, $252142 \mathrm{Kiev}$, Ukraine.

${ }^{1}$ N. Andrei and H. Johanneson, Phys. Lett. 100A, 108 (1984).

${ }^{2}$ H. J. de Vega and F. Woynarovich, J. Phys. A 25, 4499 (1992).

${ }^{3}$ H. J. de Vega, L. Mezincescu, and R. I. Nepomechie, Phys. Rev. B 49, 13223 (1994).

${ }^{4}$ P. Schlottmann, Phys. Rev. B 49, 9202 (1994).

${ }^{5}$ A. A. Zvyagin and P. Schlottmann, Phys. Rev. B 52, 6569 (1995).
${ }^{6}$ M. Fuji, S. Fujimoto, and N. Kawakami, J. Phys. Soc. Jpn. 65, 2381 (1996).

${ }^{7}$ B.-D. Dörfel and S. Meissner, J. Phys. A 30, 1831 (1997).

${ }^{8}$ O. Kahn, Y. Pei and Y Journaux, in: Inorganic Materials, ed. D. W. Bruce and D. O'Hare (Wiley, New York) (1992).

${ }^{9}$ S. Pati, S. Ramasesha, and D. Sen, Phys. Rev. B 55, 8894 (1997); preprint cond-mat/9704057 (1997).

10 S. Brehmer, H.-J. Mikeska and Shoji Yamamoto, J. Phys.: Condens. Matter 9, 3921 (1997).

${ }^{11}$ A. K. Kolezhuk, H.-J. Mikeska, and Shoji Yamamoto, Phys. Rev. B 55, R3336 (1997).

12 T. Fukui and N. Kawakami, Phys. Rev. B 55, R14709 (1997)

13 T. Tonegawa, T. Hikihara, M. Kaburagi, T. Nishino, S. Miyashita, and H.-J. Mikeska, preprint (1997).

14 T. Fukui and N. Kawakami, preprints cond-mat/9710082, 9711061 (1997).

${ }^{15}$ H. Niggemann, G. Uimin, and J. Zittartz, preprint condmat/9703206 (1997).

${ }^{16}$ M. Fannes, B. Nachtergaele, and R. F. Werner, Europhys. Lett. 10, 633 (1989).

${ }^{17}$ A. Klümper, A. Schadschneider, and J. Zittartz, J. Phys. A 24, L955 (1991).

18 M. Fannes, B. Nachtergaele, and R. F. Werner, Commun. Math. Phys. 144, 443 (1992).

19 A. Klümper, A. Schadschneider, and J. Zittartz, Z. Phys. B 87, 281 (1992); Europhys. Lett. 24, 293 (1993);

${ }^{20}$ C. Lange, A. Klümper, and J. Zittartz, Z. Phys. B96, 267 (1994).

${ }^{21}$ H. Niggemann and J. Zittartz, Z. Phys. B 101, 289 (1996).

${ }^{22}$ Gang Su, Phys. Lett. 213A, 93 (1996).

23 A. K. Kolezhuk and H.-J. Mikeska, Phys. Rev. B 56, 11380 (1997).

${ }^{24}$ I. Affleck, T. Kennedy, E. H. Lieb and H. Tasaki, Phys. Rev. Lett. 59, 799 (1987); Commun. Math. Phys. 115, 477 (1988).

25 A. Schadschneider and J. Zittartz, Annalen der Physik 4, 157 (1995).

${ }^{26}$ K. Totsuka and M. Suzuki, J. Phys.: Condens. Matter 7, 1639 (1995).

${ }^{27}$ U. Neugebauer and H.-J. Mikeska, Z. Phys. B 99, 151 (1996).

${ }^{28}$ S. Brehmer, H.-J. Mikeska, and U. Neugebauer. J. Phys.: Condens. Matter 8, 7161 (1996).

${ }^{29}$ S. Brehmer, A. K. Kolezhuk, H.-J. Mikeska, and U. Neugebauer, preprint cond-mat/9710114 (1997).

${ }^{30}$ M. Oshikawa, J. Phys. Condens. Matter 4, 7469 (1992).

31 A. A. Nersesyan and A. M. Tsvelik, Phys. Rev. Lett. 78, 3939 (1997). 


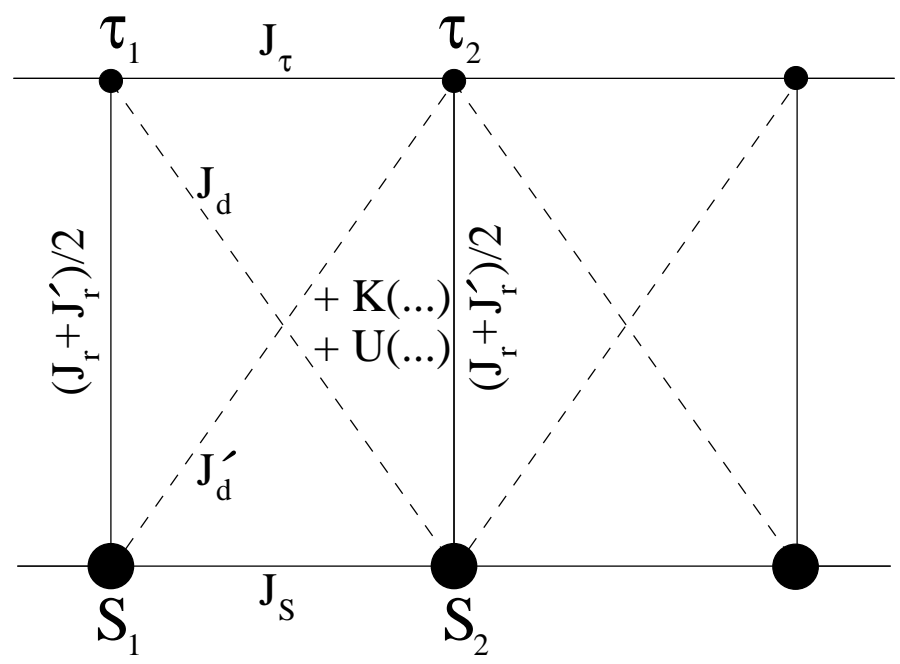

FIG. 1. Mixed spin ladder with the $S=\frac{1}{2}$ upper leg and $S=1$ lower leg as described by the model (1).

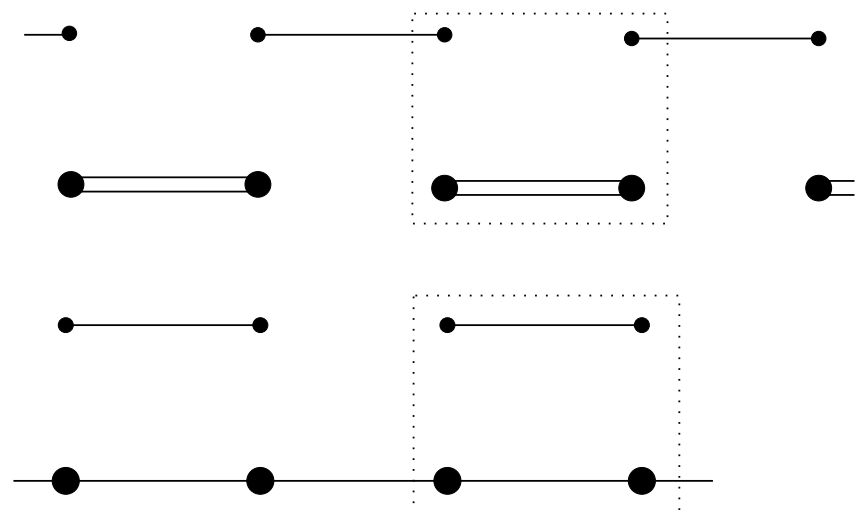

(a)

(b)
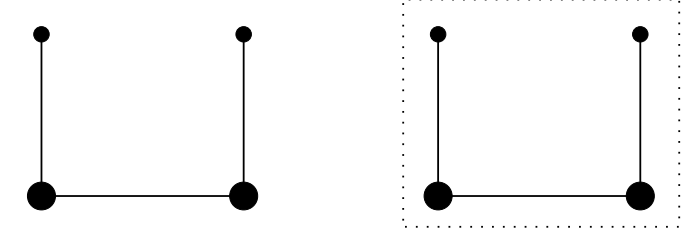

(c)

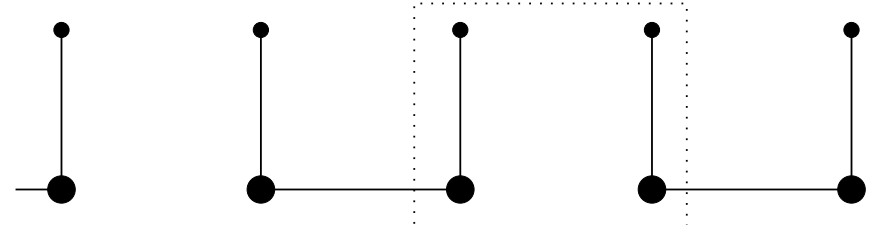

(d)

FIG. 2. A couple of VBS states which can be obtained as limiting cases of the $2 \times 4$ and $2 \times 3$ MP ansätze (3). Dotted box corresponds to the matrix product $g^{L} g^{R}$. 